\title{
Symbolic Distance Measurements Based on Characteristic Subspaces
}

\author{
Marcus-Christopher Ludl \\ Austrian Research Institute for Artificial Intelligence, Vienna
}

\begin{abstract}
We introduce the subspace difference metric, a novel heterogeneous distance metric for calculating distances between points with both continuous and (unordered) categorical attributes. Our approach is based on the computation and comparison of characteristic subspaces (i.e. contexts) for each of the symbols and can be viewed as a generalization of the well-known value difference metric.

Subsequently, as one possible extension, we propose a linearization of the computed symbolic distances by multidimensional scaling, thereby mapping a set of symbols onto the interval $[0,1]$. Thus, even algorithms, which have originally been designed for usage with continuous attributes (e.g. clustering algorithms like k-means), may be applied to datasets containing discrete attributes, without having to adapt the algorithm itself.

Finally, we evaluate the proposed metric and the linearization in quantitative and qualitative settings and exemplify the applicability in clustering domains.
\end{abstract}

\section{Introduction and Motivation}

Many inductive algorithms in machine learning and data mining make strict assumptions on the attribute types of the database. On the one hand, algorithms for dealing with continuous data may naturally utilise nearness measurements and exploit the metric properties of the instance space. On the other hand, however, restricting a knowledge structure to categorical data allows for more "exact" induction methods (like association rule or functional dependency mining), because, intuitively, categorical data does not contain that kind of inherent "fuzziness" which continuous data usually exhibits.

Many learning algorithms, most notably instance based techniques, neural networks and some of the most widely used clustering methods, necessitate all attribute types to be continuous - in these cases categorical or nominal data or missing values can often not be handled appropriately. To overcome these difficulties, in the context of classication learning, the value difference metric (VDM, refer to [SW86] or [CS93]) or one of its generalizations [WM97] has been used to good effect. Furthermore some interesting approaches for clustering heterogeneous data (i.e. datasets with mixed continuous and categorical attributes) have been published in the recent years. 
Some of these novel approaches can be regarded as self-contained methods for clustering: E.g., GKR00] and [ZFCH00] describe iterative clustering approaches based on dynamical systems, [SCC00] use a generalized notion of entropy, GRS00] propose a concept of links to measure similarity and [GGR99] introduce a summarization-based algorithm. Additionally, extensions for some well-known clustering algorithms have been developed: E.g., NH98 describe incremental and sparse variants of the EM algorithm and [GRB99] develop a discrete KMeans algorithm.

Whereas the problem of transforming continuous attributes to discrete types for the application of symbolic algorithms is well-known and usually termed discretization, none of the aforementioned methods solves the inverse problem: transforming discrete symbols to ordered (continuous or nominal) types. They either represent complete self-contained clustering procedures with little or no affinity to any continuous clustering scheme or require a rewriting of the distance metric used by the clusterer.

In this paper we propose the heterogeneous subspace difference metric (HSDM), a novel heterogeneous distance metric for computing distances between points with both continuous and categorical attributes. The basic idea is the computation and comparison of characteristic subspaces for each of the symbols, thus, the HSDM can be viewed as a generalization of the HVDM (heterogeneous value difference metric).

Furthermore, for usage as a pre-processing step, we propose a linearized extension of the SDM component, the linearized subspace difference metric (ISDM), which induces a strict ordering of the involved symbols. For this task we make use of multidimensional scaling (MDS) to map a set of symbols onto a onedimensional scale, given high-dimensional distance measurements calculated by the SDM. Thus, even algorithms, which have originally been designed for usage with continuous attributes, may be applied to datasets containing discrete attributes, without having to adapt the algorithm itself.

\section{Categorical Metrics}

As GGR99 note, distance functions on categorical attributes are not naturally defined, because it is difficult to reason that, e.g., "one color is 'like' or 'unlike' another color in a way similar to real numbers." This is due to the fact that (unordered) categorical attributes typically do not contain any information other than the symbols themselves. Whereas with continuous numbers various calculations and pairwise comparisons can be performed, usually all we can say about two colors, is whether they are equal or not.

\footnotetext{
${ }^{1}$ Some induction algorithms can be viewed as being able to discretize "on the fly" (e.g. C4.5), however also several methods for automatic pre-discretization have appeared in print: DKS95] give an excellent overview, while Lud00 present an unsupervised multivariate approach.
} 


\subsection{Overlap and VDM}

This, then, is also the most widely used (e.g. AKA91]) method for comparing two symbols: For calculating the distance between two instances with mixed continuous and categorical values, the following heterogeneous euclidean overlap metric (HEOM, refer to WM97]) is used:

$$
\operatorname{HEOM}(\boldsymbol{x}, \boldsymbol{y})=\sqrt{\sum_{i=1}^{m} d_{i}\left(x_{i}, y_{i}\right)^{2}}
$$

Here, $\boldsymbol{x}$ and $\boldsymbol{y}$ are instance vectors, $m$ is the number of attributes and $d_{i}$ is the following function:

$$
d_{i}(x, y)= \begin{cases}1 & \text { if } x \text { or } y \text { is missing } \\ \text { overlap }(x, y) & \text { if attribute } A_{i} \text { is categorical } \\ \frac{|x-y|}{\text { range }_{i}} & \text { otherwise }\end{cases}
$$

The following simple overlap function is used:

$$
\operatorname{overlap}(x, y)= \begin{cases}0 & \text { if } x=y \\ 1 & \text { otherwise }\end{cases}
$$

Clearly, the HEOM is overly simplistic in handling categorical attributes and although it may be appropriate in some cases, its use can lead to poor performance CS93.

A more sophisticated alternative, the value difference metric (VDM), introduced by [SW86], in most cases provides a better distance measurement for categorical attributes: Basically, it consists of considering two symbols to be similar, if they make similar predictions. The following definition is a simplified version without weighting terms Dom96:

$$
S V D M(x, y)=\sum_{i=1}^{c}\left|p\left(c_{i} \mid x\right)-p\left(c_{i} \mid y\right)\right|^{q}
$$

where $c$ is the number of classes, $p\left(c_{i} \mid x\right)$ is the conditional probability that the output class is $c_{i}$ given the input value $x$ and $q$ is a constant (usually 1 or 2). This categorical distance function can then be used as a replacement for the overlap function, yielding the heterogeneous value difference metric (HVDM, refer to [WM97]).

As [Dom96] note, the SVDM "attenuates [the problem of sensitivity to irrelevant attributes] for symbolic attributes, as long as a large number of examples is available, $[\ldots]$ due to the fact that by definition $p\left(c_{i} \mid x_{j}\right)$ will be roughly the same for all values $x_{j}$ of an irrelevant attribute, leading to zero distance between them." However, the SVDM is obviously only applicable in cases, where class values are available for all instances (e.g. classification tasks). 


\subsection{The Subspace Difference Metric (SDM)}

In situations, where a class attribute is not readily available (e.g. unsupervised learning), the VDM obviously cannot be used by definition. In such cases one might be tempted to fall back on the simpler overlap function to compute heterogeneous distances. With datasets, where the majority of attributes is continuous, this procedure might be appropriate - or at least not too harmful -, but when many or all of the attributes are of categorical type, much information about the distribution of the symbols will be lost thereby.

Rethinking the first paragraph in section 2, we realize that it is not exactly true. Actually, we do have more information about the symbols of a categorical attribute: we know (or can compute) the distribution of each symbol within the instance space, i.e. we know what values in other attributes each symbol co-occurs with. And this is exactly the information that we need to be able to argue that, say, the color red is more similar to orange than to blue.

Intuitively, to be able to argue about the pairwise similarity of two symbols, we have to make the assumption that the symbols do not exactly partition the domain space of another attribute. E.g. to be able to say that red is more similar to orange than to blue, we could argue that there are more instances in the dataset which can be red or orange than there are instances which can be red or blue. I.e. the context, in which the color red occurs overlaps more with the context of the color orange than with that of the color blue.

Characteristic Subspaces. The ideal tool for computing the overlaps between such contexts would be characteristic rules or the characteristic subspaces induced by them. Formally:

Definition 1. Let $A_{i}$ represent the $i$-th attribute $(i \in\{1, \ldots, m\})$ and $V_{i}$ be a set of values from the domain of attribute $i$. A characteristic rule is an implication rule of the form

$$
A_{i}=x \rightarrow A_{1} \in V_{1} \wedge \cdots \wedge A_{m} \in V_{m}
$$

where the attribute $A_{i}$ does not occur on the right hand side. A characteristic rule is said to hold with confidence $p$, formally

$$
A_{i}=x \rightarrow_{p} A_{1} \in V_{1} \wedge \cdots \wedge A_{m} \in V_{m}
$$

if, with probability at least $p$, whenever condition $A_{i}=x$ holds for an instance, the right hand side holds as well.

A characteristic rule is basically an association rule, where the left hand side is restricted to only one condition. $V_{i}$ is a discrete set of values, if $A_{i}$ is categorical, and a continuous interval, if $A_{i}$ is continuous.

Usually characteristic rules are constructed for values of a designated class attribute, we may, however, induce such rules for symbols from any categorical attribute. Thereby we obtain information about the context in which a certain symbol occurs - the subspaces induced by these rules will in the following be 
called characteristic subspaces. Intuitively, a characteristic subspace gives an extensional description of a symbol and by comparing these spaces we could argue about the similarity of the symbols.

Projected Characteristic Subspaces. Unfortunately, computing such characteristic subspaces with high confidences would necessitate running a characteristic rule induction algorithm for each single symbol, which is obviously computationally infeasible. For rules with high confidence it would, e.g., not suffice to compute the projections of a symbol onto each of the other attributes and combine them, because the cartesian product of two or more highly probable regions in different attributes need not necessarily be highly probable as well, formally:

$$
A_{i}=x \rightarrow_{p}\left(A_{j} \in V_{j}\right), A_{i}=x \rightarrow_{p}\left(A_{k} \in V_{k}\right) \nRightarrow A_{i}=x \rightarrow_{p}\left(A_{j} \in V_{j} \wedge A_{k} \in V_{k}\right)
$$

However, in our case, we may relax the requirements, in that we actually need not construct full characteristic subspaces. For the comparison of two symbols it does suffice to compare the projections onto each of the other attributes. We call the resulting spaces projected characteristic subspaces (pc-subspaces).

Definition 2. With $D_{i}$ representing the domain of attribute $A_{i}$, let the discrete domain $\bar{D}_{i}$ be defined as follows:

$$
\bar{D}_{i}= \begin{cases}D_{i} & \text { if attribute } A_{i} \text { is categorical } \\ \{1, \ldots, d\} & \text { if attribute } A_{i} \text { is continuous, for } d \in \mathbb{N}\end{cases}
$$

To construct the discrete domains of the attributes, we have to discretize the continuous attributes by some simple unsupervised discretization method. Preferably we should use equal-width discretization for this task, because this method visualizes the distribution of the values within the interval (in the experiments we chose $d=6$ as standard setting).

Definition 3. Let $p_{j}$ be the discrete probability density of the values from the discrete domain $\bar{D}_{j}$ of attribute $j$ and let $p_{j, A_{i}=x}$ be this discrete probability density under the condition $A_{i}=x$ (i.e. $p_{j, A_{i}=x}(y)=p_{j}\left(y \mid A_{i}=x\right)$ ). Then the probabilistic projected characteristic subspace (ppc-subspace) of the symbol $x$ in attribute $A_{i}$ is defined as the collection of all densities $p_{j, A_{i}=x}$ for $j \neq i$.

Definition 4. With $m$ being the number of attributes, $x$ and $y$ symbols from a categorical attribute $A_{i}$ and $q \in \mathbb{N}$, the subspace difference metric (SDM) is defined as follows:

$$
S D M_{i}(x, y)=\sum_{\substack{j=1 \\ j \neq i}}^{m} \sum_{v \in \bar{D}_{j}}\left|p_{j, A_{i}=x}(v)-p_{j, A_{i}=y}(v)\right|^{q}
$$


For convenience and ease of computation, the ppc-subspace of a symbol can be written as a matrix of dimensions $m \times s$, with $m$ being the number of attributes and $s:=\max \left\{\left|\bar{D}_{1}\right|, \ldots,\left|\bar{D}_{m}\right|\right\}$. The positions in this matrix are the respective conditional probabilities; columns having less than $s$ symbols are filled with zeros up to $s$ elements. That way, the calculation of a symbolic distance can be seen as a matrix operation.

Finally, plugging the SDM into the heterogeneous metric, where previously the (S)VDM or the overlap function was used, yields the HSDM:

Definition 5. With $m$ being the number of attributes and $q \in \mathbb{N}$ the heterogeneous subspace difference metric (HSDM) of two instances $\boldsymbol{x}$ and $\boldsymbol{y}$ is defined as follows:

$$
\operatorname{HSDM}(\boldsymbol{x}, \boldsymbol{y})=\sum_{i=1}^{m} d_{i}\left(x_{i}, y_{i}\right)^{q}
$$

with the distance function $d_{i}$ defined heterogeneously as follows:

$$
d_{i}(x, y)= \begin{cases}1 & \text { if } x \text { or } y \text { is missing } \\ \operatorname{SDM}_{i}(x, y) & \text { if attribute } A_{i} \text { is categorical } \\ \frac{|x-y|}{\text { range }_{i}} & \text { otherwise }\end{cases}
$$

Complexity. Due to the fact that the ppc-subspace of a symbol can be written as a matrix of dimensions $m \times s$ (section 2.2) the comparison of two symbols is linear in the number of attributes $m$ and in the maximum number of unique symbols $s$ in any categorical attribute. Thus, the computation of a heterogeneous distance between two instances is, in the worst case, $O\left(\mathrm{~m}^{2} s\right)$. Unfortunately, however, the explicit pre-computation of the ppc-matrices would take time $O\left(m^{2} n^{2}\right)(n$ being the number of instances), yielding $O\left(n^{2}\right)$ complexity for the computation of a dissimilarity matrix as well.

By means of a hashtable, however, many of the computations can be simplified. If, e.g., a complete dissimilarity matrix is to be computed, the ppc-subspaces of all symbols can be pre-computed by scanning through all instances and recording each co-occurrence of two symbols $x$ and $y$ in two attributes $a_{1}$ and $a_{2}$. That way, the ppc-subspace of a symbol is only implicitly represented by all associations (along with their counts) for the according symbol stored in the hashtable. This pre-computation step basically consists of linearly scanning the database and thus can be realized in time $O\left(m^{2} n\right)$.

\section{Linearization by Multidimensional Scaling}

One possibility for modularly implementing the SDM in a learning algorithm is the HSDM as defined above. However, this necessitates (at least partly) a rewriting of the involved algorithm. Unfortunately, this is not always possible. Also, it may prove advantageous for subsequent quantitative data mining techniques to have available nearness information between the symbolic values. 
A transformation of the categorical attributes to continuous types in a preprocessing step could thus be a possible solution. However, we have to be aware of the fact that this task can not always be performed well and that in some cases the simple overlap metric might indeed be more appropriate.

We now extend the SDM to a linearized version, the linearized subspace difference metric (ISDM). We accomplish this by applying multidimensional scaling (MDS) methods to the distance measurements induced by the SDM.

MDS is typically used for computing representative data points for highdimensional data (which should, e.g., be visualized) or proximity data (sometimes even incomplete) in a suitable low-dimensional space, such that the distances between the projected data points match the original distance values as faithfully as possible. The basic idea consists of minimizing a cost function, usually stress, raw stress, strain or something similar. The original algorithm for minimizing stress can be found in Kru64], Sammon mapping, also one of the oldest approaches, can be found in Sam69. dLJ77 describe the widely used majorization method for MDS and KB97] present an application of deterministic annealing to the problem.

In our case the task can be defined as projecting data points of $m \times s$ dimensions (see section 2.2) onto a continuous scale, i.e. one dimension. For this projection we applied a simple gradient descent approach, minimizing raw stress.

The usual problem of finding good initial configurations applies here as well: Applying a strict gradient descent algorithm to a one-dimensional configuration can only move the points around a bit, but cannot change their relative order. However, because the target space is only one-dimensional, we have available a few canonical configurations, which should work reasonably well as starting points:

For a categorical attribute $A_{i}$ we construct $\left|\bar{D}_{i}\right|$ different initial configurations by using one of the symbols $x_{j}$ as the point of origin and placing the other symbols at positions which conform to their distances from $x_{j}$. We then run the gradient descent algorithm on each of the $\left|\bar{D}_{i}\right|$ configurations and accept the final configuration with the lowest raw stress as the resulting projection.

\section{Experimental Evaluation}

\subsection{Quantitative Results}

In a first set of experiments we exemplify the applicability of our approach in a clustering setting: We chose several datasets with varying characteristics from the UCI Machine Learning Repository and from the Esprit Project StatLog.

Apart from hayes-roth and postoperative (patient), all datasets had both continuous and categorical source attributes. As reference clusterings (for the calculation of recall) we used the class label\$2; in case of the servo dataset,

\footnotetext{
${ }^{2}$ It is reasonable to assume that the existing class labels correlate with well-defined subspaces within the instance space. There is, however, no theoretical argument corroborating this assumption.
} 
where the target variable is continuous, we chose to pre-discretize the target by equal-width into 6 classes (i.e. clusters).

The synthetic dataset had five attributes: The attributes 1, 3 and 4 were continuous (ranges $[1,100],[1,50]$ and $[1,100]$, respectively), while the attributes 2 and 5 were symbolic: $\{A, B\}$ and $\{w, y, o, r\}$. The following rules held for the class attribute: $\left(a_{2}=A \wedge a_{3} \geq 20 \rightarrow\right.$ class $\left.=1\right),\left(a_{2}=B \wedge a_{3} \leq 15 \rightarrow\right.$ class $\left.=2\right)$, $\left(a_{5}=w \rightarrow\right.$ class $\left.=3\right)$ and $($ else $\rightarrow$ class $=4)$.

As a clusterer we used the k-means implementation pam KR90], which relies on the pre-computation of a dissimilarity matrix before clustering, from the cluster package of the freely available and widely used statistics software $\mathrm{R}$. We compared three different methods: In the first two runs we used daisy $y^{3}$ and the HSDM 4 , respectively, for the computation of the dissimilarity matrix before applying pam. In a third run we used the ISDM to transform the symbols into real numbers and applied pam to the continuous dataset.

For each clustering thus generated we computed two different quality measures: On the one hand we used the silhouette coefficient from [KR90] as an internal measure. On the other hand we used recall (refer to [LW02]) to compare the resulting clustering to the reference clustering. Table 1 shows the results.

Table 1. Comparative clustering results for various datasets. Shown are the silhouette widths ("silh") and recall values (tolerance $=0.5$ ) of k-means clusterings. The highest numbers are printed in bold (no significance test applied).

\begin{tabular}{|c|c|c|c|c|c|c|}
\hline data & ws & cols & classes & $\begin{array}{c}\text { daisy } \\
\text { silh. recall }\end{array}$ & $\begin{array}{c}\text { hsdm } \\
\text { silh. recall }\end{array}$ & $\begin{array}{c}\text { lsdm } \\
\text { silh. recall }\end{array}$ \\
\hline post & 90 & 8 & 3 & $0.18 \quad 0.24$ & $0.18 \quad 0.24$ & $\mathbf{0 . 2 5} \quad 0.2$ \\
\hline ha & 132 & 4 & & $\begin{array}{ll}0.23 & 0.00\end{array}$ & $0.29 \quad 0.00$ & $\begin{array}{ll}0.33 & 0.00\end{array}$ \\
\hline & & 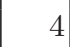 & 6 & $0.14 \quad 0.12$ & 0.540 .16 & $.38 \quad 0.13$ \\
\hline & 200 & 5 & 4 & $0.25 \quad 0.10$ & $0.40 \quad \mathbf{0 . 1 9}$ & 0.440 .05 \\
\hline & 27 & 13 & 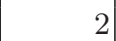 & $0.26 \mathbf{0 . 6 8}$ & $0.24 \quad 0.56$ & $\begin{array}{ll}0.38 & 0.40\end{array}$ \\
\hline$c x$ & 690 & 15 & 2 & $\begin{array}{lll}0.17 & 0.64\end{array}$ & $0.18 \quad 0.63$ & 0.19 \\
\hline
\end{tabular}

As can be seen, the HSDM and/or the ISDM in most cases allow for better clusterings: In all cases the silhouette width is higher, which is not really surprising, given that the HSDM naturally allows for more accurate distance measurements than the HEOM - a fact that typically has a positive impact on internal quality measures. We have illustrated this consideration by 2-dimensional cluster plots in figure 1 .

\footnotetext{
${ }^{3}$ Basically, daisy relies on a slightly more sophisticated HEOM to calculate heterogeneous distances by using weighting factors for each attribute. daisy is described in detail in KR90.

4 To be able to use the same format for the dissimilarity matrix we implemented the HSDM in pure R-code (no Fortran), which did not use hashtables (refer to section 2.2.). Running times are therefore not comparable.
} 

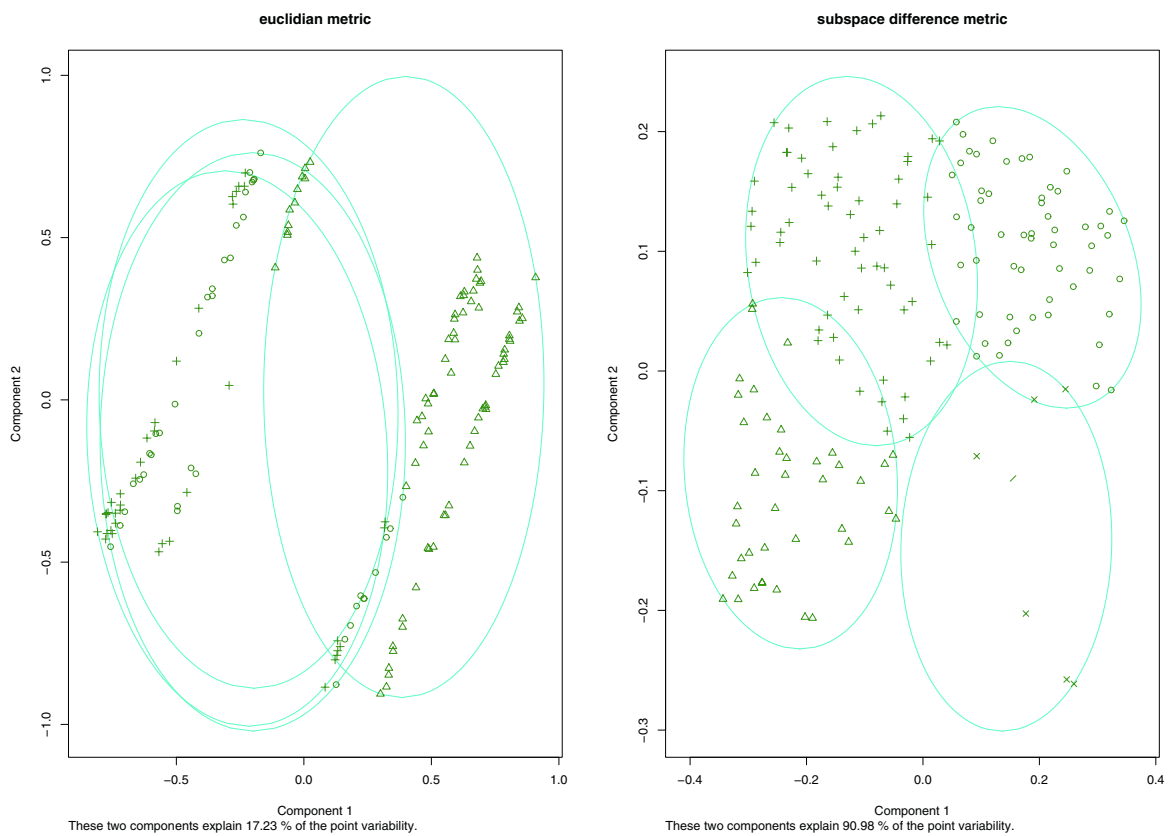

Fig. 1. Cluster plots (mapped to two dimensions) for the synthetic dataset (see text), clustered by k-means (4 centers) in conjunction with the HEOM (daisy) and the HSDM, respectively.

However, in most cases also the recall values of SDM-clusterings increase. From these results it is reasonable to assume that a lower clustering quality is largely due to an inappropriate handling of symbolic attributes.

It has to be noted that the HEOM achieved a better recall rating in one case and did not do much worse in two others. As mentioned in section 3, this might be due to the fact that in some cases - especially when the symbols have no inherent canonical order - inducing an artificial order instead of using 0-1equality might actually be detrimental to clustering quality.

\subsection{Qualitative Results}

In a second experiment we wanted to investigate whether the linearized SDM $l S D M$ is able to capture canonical orderings within symbolic attributes, i.e. whether the concept of characteristic subspaces can indeed reflect intuitively obvious orderings and whether these orderings are preserved by scaling the multidimensional information down to only one dimension.

For this test we chose a more complex, but rather small dataset: Pittsburgh Bridges (from the UCI repository) is a "design domain" rather than a classification domain, where 5 design descriptions need to be predicted based on 7 
specification properties. The dataset has 108 instances with 13 attributes and few missing values. We chose to eliminate the two "identifying" attributes (id and location).

In table 2 we list the orderings induced by applying the ISDM to the discretized version of the dataset. The correct (or intended) orderings of most of the attributes are know, because they have been discretized from numeric values - e.g. the attribute erected reflects time epochs from 1818 to 1986. Note that even the attributes lanes (which contains 4 nominal values) was treated as discrete in this context.

Table 2. The induced orderings of the symbolic values in the Pittsburgh Bridges domain. Only symbolic attributes with a canonical order are shown, the induced values for the missing value-symbol are omitted. Note: Some numbers are presented in reverse order - this, of course, does not affect the resulting metric.

\begin{tabular}{|l|l|l|}
\hline attribute & correct order & induced order \\
\hline erected & crafts & crafts $(2.93)$ \\
& emerging & emerging (1.28) \\
& mature & mature (0.64) \\
& modern & modern (0.00) \\
\hline length & short & short (1.83) \\
& medium & long (1.00) \\
& long & medium (0.49) \\
\hline lanes & 1 & $1(4.22)$ \\
& 2 & $2(2.16)$ \\
& 4 & $4(1.09)$ \\
& 6 & $6(0.00)$ \\
\hline span & short & short (0.00) \\
& medium & medium (1.96) \\
& long & long (2.72) \\
\hline
\end{tabular}

As can be seen, linearizing the symbols by the ISDM can re-construct the former orderings in all but one attribute, where the order of medium and long is inverted. Note that we only list the results for attributes with a known canonical ordering here and omit results for attributes like, e.g., purpose (with the values walk, aqueduct, $r r$ and highway).

\section{Summary and Discussion}

We have presented the novel heterogeneous metric HSDM for computing distances between points with categorical or mixed-continuous-categorical attribute values. The approach is based on the concept of (probabilistic projected) characteristic subspaces, which can also be viewed as a generalization of the well-known value difference metric. Additionally, we have introduced the idea of linearizing the high-dimensional distance matrices by multidimensional scaling, thereby 
yielding the ISDM, a reverse transformation to discretization: encoding symbols by continuous numbers.

We have exemplified in a clustering setting that the HSDM most often yields better results in terms of internal (silhouette width) and external (recall) clustering quality measures than the widely used HEOM. Finally, in a qualitative setting, we have shown that the ISDM yields good transformations of the involved symbols into real numbers, especially when an obvious canonical order among the symbols exists.

It has to be noted that the transformation does not always produce superior (quantitative or qualitative) results. Especially in cases, when no intuitively obvious order among the symbols can be found, the naive overlap function yields slightly better clusterings. This is obviously due to the fact that in such cases, the computation of 0-1-equality is "as good as it gets" and any artificial ordering of the symbols might deteriorate the results. A combination of both approaches in a single metric is one of our future research topics.

Furthermore, the mapping of the high-dimensional subspace data to one continuous dimension is obviously a crucial step in our algorithm. Using a more sophisticated method for MDS might further improve the final transformation and thus is also one of our topics for future research.

Finally, as one reviewer pointed out, it may be expected that the SDM should lend itself well for application in instance based learning tasks (nearest neighbor classification). We have chosen to evaluate the proposed metric in a clustering setting mainly because - unlike the VDM - it was intended to be used without a designated class attribute and will, eventually, be part of a novel clustering algorithm. Evaluating the SDM in IBL settings is a valuable suggestion, which we plan to tackle in the near future.

\section{Acknowledgements}

This research is supported by the Austrian Fonds zur Förderung der Wissenschaftlichen Forschung (FWF) under grant no. P12645-INF. The Austrian Research Institute for Artificial Intelligence acknowledges basic financial support by the Austrian Federal Ministry for Education, Science, and Culture.

We would like to thank Peter Filzmoser for invaluable discussions and ideas. We would also like to thank the anonymous reviewers for their helpful comments and suggestions.

\section{References}

AKA91. D.W. Aha, D. Kibler, and M.K. Albert. Instance-based learning algorithms. Machine Learning, 6:37-66, 1991.

CS93. S. Cost and S. Salzberg. A weighted nearest neighbor algorithm for learning with symbolic features. Machine Learning, 10:57-78, 1993.

DKS95. J. Dougherty, R. Kohavi, and M. Sahami. Supervised and unsupervised discretization of continuous features. In Prieditis A. and Russell S., edi- 
tors, Proceedings of the $12^{t} h$ International Conference on Machine Learning (ML'95), San Francisco, CA, 1995. Morgan Kaufmann.

dLJ77. de Leeuw. J. Applications of convex analysis to multidimensional scaling. In J. Barra, F. Brodeau, G. Romier, and B. van Cutsem, editors, Recent Developments in Statistics, pages 133-145. North Holland Publishing Company, The Netherlands, 1977.

Dom96. P. Domingos. Unifying instance-based and rule-based induction. Machine Learning, 24(2):141-168, August 1996.

GGR99. V. Ganti, J. Gehrke, and R. Ramakrishnan. Cactus - clustering categorical data using summaries. In Knowledge Discovery and Data Mining, pages 73-83, 1999.

GKR00. D. Gibson, J. Kleinberg, and P. Raghavan. Clustering categorical data: An approach based on dynamical systems. VLDB Journal (Very Large Databases), 8(3-4):222-236, 2000.

GRB99. S.K. Gupta, K.S. Rao, and V. Bhatnagar. K-means clustering algorithm for categorical attributes. In Data Warehousing and Knowledge Discovery, pages 203-208, 1999.

GRS00. S. Guha, R. Rastogi, and K. Shim. ROCK: A robust clustering algorithm for categorical attributes. Information Systems, 25(5):345-366, 2000.

KB97. H. Klock and J.M. Buhmann. Multidimensional scaling by deterministic annealing. In Energy Minimization Methods in Computer Vision and Pattern Recognition, pages 245-260, 1997.

KR90. L. Kaufman and P.J. Rousseeuw. Finding Groups in Data: An Introduction to Cluster Analysis. Wiley, NY, USA, 1990.

Kru64. J. Kruskal. Multidimensional scaling by optimizing goodness of fit to a nonmetric hypothesis. Psychometrika, 29(1):1-27, March 1964.

Lud00. M.-C. Ludl. Relative unsupervised discretization of continuous attributes. Master's thesis, Institut für Medizinische Kybernetik und Artificial Intelligence, University of Vienna, Austria, 2000.

LW02. M.-C. Ludl and G. Widmer. Towards a simple clustering criterion based on minimum length encoding. In Proceedings of the $13^{\text {th }}$ European Conference on Machine Learning (ECML-02), pages 258-269, Helsinki, Finland, 2002. Springer Verlag.

NH98. R.M. Neal and G.E. Hinton. A view of the EM algorithm that justifies incremental, sparse and other variants. In M.I. Jordan, editor, Learning in Graphical Models. Kluwer, 1998.

Sam69. J.W. Sammon. A nonlinear mapping for data structure analysis. IEEE Trans. Comp., C-18(5):401-409, 1969.

SCC00. D.A. Simovici, D. Cristofor, and L. Cristofor. Generalized entropy and projection clustering of categorical data. In Proceedings of the $4^{\text {th }}$ European Conference on Principles and Practice of Knowledge Discovery in Databases (PKDD2000). Springer, 2000.

SW86. C. Stanfill and D. Waltz. Toward memory-based reasoning. Communications of the ACM, 29:1213-1228, 1986.

WM97. D.R. Wilson and T.R. Martinez. Improved heterogeneous distance functions. Journal of Artificial Intelligence Research, 6:1-34, 1997.

ZFCH00. Y. Zhang, A.W. Fu, C.H. Cai, and P.-A. Heng. Clustering categorical data. In $I C D E$, page $305,2000$. 